\title{
Motivational interviewing as substance treatment for college students
}

\begin{abstract}
With rising severity of substance use on college campuses, 1,2 college counseling centers should prepare to provide effective substance abuse treatments. Motivational interviewing is a therapeutic approach developed through research and clinical practice with addiction. $3 \mathrm{MI}$ is more than a simple set of techniques because of its humanistic framework which grounds MI in a person-centered foundation.3 College counseling centers looking for substance abuse treatment models should consider MI as a well-researched and evidence-based therapy model.3-7 The emphasis of therapeutic alliance has strong potential to quickly connect with clinicians' previous knowledge of relationship building training. 3 Its change-oriented therapy process delivers a much needed therapeutic focus in the treatment of college students' substance abuse. Our review illustrates the rich and detailed information of MI in this book. College counseling center could easily access this book to evaluate the best use of MI in treatment process.
\end{abstract}

Keywords: college students, substance abuse, college counseling centers, motivational interviewing
Volume 3 Issue 6 - 2017

\author{
Yuh-Jen Guo, Shu-Ching Wang, Katie M \\ Immerfall \\ St. Cloud State University, USA
}

\begin{abstract}
Correspondence: Yuh-Jen Guo, Clinical Mental Health Counseling Program of Community Psychology, Counseling and Family Therapy at St. Cloud State University, 210 Education Building, 720 Fourth Avenue South, St. Cloud, Minnesota 56301 USA, Tel 320-308-4865, Fax 320-308-3216, Email mguo@stcloudstate.edu
\end{abstract}

Received: August 08, 2017 | Published: August 17, 2017
Abbreviations: MI, motivational interviewing; OARS, open questions-affirming-reflecting-summarizing

\section{Introduction}

Substance abuse has become a severe mental health issue among college students. ${ }^{1,8,9}$ Understanding the tremendous amount of stress arising in college life, ${ }^{1,10}$ scholars have suggested that college students are turning into a vulnerable group where substance abuse prevalence has seen a dramatic increase. ${ }^{11}$ Various types of substance abuse spread widely on college campuses. Students learn to use substances in colleges and they seek substances to relieve stress. Alcohol, nicotine, marijuana, and prescription medicines are among the substances invading college campuses. ${ }^{12-16}$ The severity of substance abuse in college students has raised scholars' attention to substance use and treatment on college campuses.

College counseling centers act as on-campus clinics, which directly provide psychotherapy to college students. Counseling centers also deliver substance abuse treatments in order to meet students' needs. ${ }^{4,5}$ Among substance abuse treatment models, a therapeutic approach most attracts the attention of mental health professionals who serve college students. ${ }^{67,14,17}$ Motivational interviewing (MI) is one such approach, which developed specifically for treating substance abuse cases. ${ }^{3}$ MI is a proven effective treatment approach for substance users. ${ }^{3-7}$ Some college counseling centers have implemented MI to help college students who have developed substance use addiction. It is crucial to examine the MI approach for potential use in college counseling centers to prevent the high prevalence of substance abuse on college campuses.

\section{Discussion}

Motivational interviewing (MI) was developed by William $\mathrm{R}$ Miller \& Stephen Rollnick ${ }^{3}$ out of their research and clinical practice in addiction therapy. ${ }^{3} \mathrm{MI}$ underwent years of development, and a recent edition of MI book was published in 2013. The third edition of Motivational Interviewing: Helping People Change provides the newest MI framework with concepts, therapy processes, and clinical examples. ${ }^{3}$ We want to briefly discuss the conceptual framework and therapeutic processes of MI described in this book. Our hope is that such discussion may provide necessary information for counseling centers looking for substance abuse treatment approaches. In the battle with the wide spreading substance abuse on college campuses, MI could be an effective tool for college counseling centers to assist students with substance abuse.

\section{Have "change" in mind}

The layout of this new edition starts with a brief introduction of MI. The first and foremost discussion about MI is its focus on "change." The core therapeutic effectiveness is grounded on the ability of facilitating individuals' motivation to change and execution of strategies leading to behavior change. Miller and Rollnick describe the existence of "change" in daily conversation, and how clinicians in mental health and health care fields should pay attention to the opportunities for engaging in those "change" conversations. ${ }^{3}$ Miller and Rollnick suggest clinicians avoid the directing and following styles in conversation. ${ }^{3}$ Directing style has clinicians give advice and instruction and following style has clinicians passively stay behind clients' stories. The guiding style asks clinicians to effectively listen to clients' desires to change but also actively provide expert opinions to enhance the clients' determination to change. The road to change is illustrated as the goal of MI and the effective style is essential for achieving such goal.

\section{Spirit: the foundation of MI}

While evidence-based practice is established as the standard in clinical practice, Miller \& Rollnick ${ }^{3}$ take one chapter to discuss the spirit of MI. ${ }^{3}$ This discussion reflects the importance of therapeutic relationship versus overemphasis on techniques. In a book written for health care practitioners, Miller, Rollnick \& Butler ${ }^{18}$ present 
MI in a technical process with abundant examples to teach the MI conversation style. ${ }^{18}$ The discussion of MI spirit is short and brief in their 2008 book, which looks more like guidelines than essential elements. Miller and Rollnick provide a well- developed version of the MI spirit with detailed explanations of its four elements:
a. Partnership
b. Acceptance
c. Compassion
d. Evocation

A clinician practicing MI must realize that MI is not a word game in which the therapeutic effectiveness relies on insertion and imposition of the clinician's choices through skillful manipulation. Miller and Rollnick portray MI spirit in a humanistic and supportive manner, which emphasizes the collaboration of the therapeutic process, empathetic understanding and commitment to clients, and the empowering of clients' strengths.

\section{From engaging to planning}

Miller \& Rollnick ${ }^{3}$ plan a four-step process in MI. The four processes begin with engaging, focusing, evoking, and end with planning. ${ }^{3}$ From there on, the book is arranged in four major parts. These parts are labeled with the names of the four processes, and each part organizes several chapters to further the discussion of concepts and techniques under each of the four processes. Although these chapters lay a map for technique seekers, there is a clear thread of MI spirit flowing beneath the concepts and practice of MI techniques. It is fair to say that the practice of MI techniques aims to execute the contents sketched in the four elements of MI spirit. The fulfillment of MI spirit leads to the success of MI processes.

Part II: Engaging: Engaging an individual who is either willing or not willing to receive substance abuse treatment is what clinicians encounter daily in their offices. The engaging process in MI stems from the MI spirit to carefully build a collaborative relationship, which is nurtured by the partnership of the MI spirit. Miller \& Rollnick first emphasize the critical role of relationship serving as the foundation of the therapeutic process. ${ }^{3}$ Literature cited by Miller \& Rollnick $^{3}$ provides support for this evidence-based practice when it indicates that quality of relationship, or therapeutic alliance, greatly contributes to the outcomes of therapy. The warning of traps, which hinder relationship building, active listening, and reflection, are given to better prepare clinicians in engaging. A technical acronym, OARS, is described as the "foundational tools" for establishing therapeutic alliance. OARS stands for: Open questions-Affirming-ReflectingSummarizing. ${ }^{3}$ These technical tools follow the MI spirit in acceptance and compassion, when clinicians are able to affirm clients' worth, and to reflect and summarize contents with understanding and commitment to clients' dilemma.

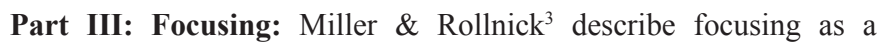
continuous process, which aims to keep the therapeutic process on track. ${ }^{3}$ Unlike the non-directive approach proposed by Carl Roger's person-centered therapy, MI pays attention to the direction of therapy. However, Miller and Rollnick do not suggest taking a directive charge to the therapeutic goals. Instead, focusing takes a collaborative process where clinicians and clients share the control and the clients' autonomy is respected and supported. Focusing is a process to help clinicians avoid the ineffective processes and traps that may not contribute to clients' change. With the focusing process starting early in the engaging process, it assists clinicians in maintaining a clear vision of the therapeutic process and guides clients to explore the direction towards their desired changes.

Part IV: Evoking: Evoking is recognized by Miller \& Rollnick ${ }^{3}$ as a unique process endorsed by MI. While engaging and focusing would be conceptualized in different ways and used in other forms of therapeutic approaches, evoking adds a new aspect and separates MI from other therapeutic approaches. ${ }^{3}$ Building upon the foundation of the relationship established through engaging and focusing, evoking starts a process to move clients toward changing current behaviors. The conversation is heard with ears tuned into clients' ambivalence towards change and the potential towards change in change talk. Clinicians work with change talk to elicit motivation to change and avoid sustain talk, which stops clients from making changes. They also utilize the OARS to engage change talk and enhance the direction towards change. Even though in a process to push towards change, evoking does respect clients' autonomy and their choices without blaming or shaming them for failure. This is also a process to provide confidence and hope to those who are clear about their direction, but lack trust in their own ability to succeed. However, according to the MI spirit, Miller \& Rollnick ${ }^{3}$ clearly depict the evoking process as a collaborative and supportive process. ${ }^{3}$ Evoking is not a one-way insertion of clinicians' intended goals into clients' action plans. It is rather a process where clinicians use the MI techniques to promote changes within clients and help them clear the roadblocks such as ambivalence, sustain talk, and lack of confidence.

Part V: Planning: The last MI process is planning, which involves composing a strategic plan on how to achieve desired change. Miller $\&$ Rollnick $^{3}$ indicate planning is a necessary process to prevent relapse and loss of motivation after clients go through engaging, focusing, and evoking. ${ }^{3}$ Clinicians assess clients' readiness for planning when observing increased change talk and reduced sustain talk. In the planning process, it calls for developing specific steps in the plan and dealing with uncertainty and reluctance with strengthened commitment. Clinicians facilitate the planning process and encourage clients to commit to execution of the plan, while they continue to provide clients support and guidance when challenges emerge during the execution of the plan. The therapeutic process continues even when clinicians have to re-visit the beginning of the four processes. There will be time when clinicians should help clients in re-engaging and re-focusing steps to align them on the track towards change.

This brief review of Miller \& Rollnick's ${ }^{3}$ most recent revision of MI intends to provide a quick reference for potential users, such as college counseling centers mentioned in this review. MI is constructed upon a supportive and collaborative therapeutic relationship, which nurtures the environment for the execution of MI processes. It is critical to realize that MI is more than a set of techniques for challenging substance users' inability and resistance to change. Implementation of $\mathrm{MI}$ in counseling centers will require proper MI training for clinicians to firmly acquire the MI spirit as the foundation for MI processes and techniques. Miller \& Rollnick ${ }^{3}$ mention the person-centered approach in relationship building and this basic therapeutic process should be mastered. ${ }^{3}$ Proper supervision has to be provided to facilitate the early stage development of clinicians when they practice MI processes and learn to work with clients under the MI framework.

\section{Conclusion}

College students' substance use increases dramatically in recent years. ${ }^{1,2,19}$ The severity and prevalence of substance use in college 
student populations adversely impact students' academic performance. We introduce the current version of Motivational interviewing (MI) by briefly reviewing the developers' book. ${ }^{3}$ The current book of MI clearly lists its processes and provides rich clinical examples for learners. MI is seemingly a simple set of techniques used to promote change in addiction, but it carries a humanistic framework which grounds MI in a person-centered foundation and allows MI to utilize the strength of therapeutic alliance in its processes

Miller \& Rollnick ${ }^{3}$ urge clinicians to think of MI beyond the stereotype of counseling techniques, and to understand the spirit of MI and how it is reflected in MI processes. ${ }^{3}$ However, MI also includes common therapeutic elements and factors in the MI spirit and processes. Clinicians with solid therapeutic training and experience could smoothly transit to the MI framework. Miller \& Rollnick devote part of their writing to the use of MI in daily life and clinical practice. ${ }^{3}$ Their effort in translating MI to be easily comprehended and reproduced in the clinicians' world makes the reading of Miller and Rollnick's book a pleasant journey of learning and understanding of MI. College counseling centers looking for substance abuse treatment models should consider MI as a well-researched and evidence-based therapy model. MI is a treatment approach with empirical support on its effectiveness as a therapy model for substance users. ${ }^{3-7}$ It is a therapeutic approach developed through research and clinical practice with addiction. ${ }^{3}$ MI constructs its foundation on relationship building. Therefore, it has strong potential to quickly connect with clinicians' previous knowledge because most clinicians received relationship building training in academic study. ${ }^{3}$ It also actively steers the therapeutic process to behavior change, a much needed focus in the treatment of college students' substance abuse. College counseling centers combating students' substance use should consider the adoption of a change-oriented treatment approach like MI. While MI approach has been thoroughly presented in Miller and Rollnick's book, college counseling centers have an easy access to this model to evaluate its fit to the centers' missions.

\section{Acknowledgements}

None.

\section{Conflict of interest}

The author declares no conflict of interest.

\section{References}

1. Anderson DS. New directions for substance-abuse prevention. Change. 2011;43(6):46-55.

2. Baldwin JA, Johnson RM, Gotz NK, et al. Perspectives of college students and their primary health care providers on substance abuse screening and intervention. $J$ Am Coll Health. 2006;55(2):115-119.
3. Miller WR, Rollnick S. Motivational interviewing: Helping people change. 3rd ed. New York: Guilford press; 2012. 482 p.

4. Hayes BG, Curry J, Freeman MS, et al. An alternative counseling model for alcohol abuse in college: A case study. $J$ Coll Counseling. 2010;13:87-96.

5. Scholl MB, Schmitt DM. Using motivational interviewing to address college client alcohol abuse. J Coll Counseling. 2009;12(1):57-70.

6. Ginter GG, Choate LH. Stage-matched motivational interventions for college student binge drinkers. J Coll Counseling. 2003;6(2):99-113.

7. Petry NM, Weinstock J, Morasco BJ, et al. Brief motivational interventions for college student problem gamblers. Addiction. 2009;104(9):1569-1578.

8. Giordano AL, Cashwell CS. Exploring the relationship between social interest, social bonding, and collegiate substance abuse. J Coll Counseling. 2014;17(3):222-235.

9. West SL, Graham CW. A survey of substance abuse prevention efforts at Virginia's colleges and universities. J Am Coll Health. 2005;54(3):185191.

10. Lunney M. Stress overload: A new diagnosis. Int J Nurs Terminol Classif. 2006;17(4):165-175.

11. Zack M, Poulos CX, Aramakis VB, et al. Effects of Drink-Stress sequence and gender on alcohol stress response dampening in high and low anxiety sensitive drinkers. Alcohol Clin Exp Res. 2007;31(3):411-422.

12. Sharma M. Binge drinking interventions among college students. $J \mathrm{Al}$ cohol Drug Educ. 2008;52(2):3-8.

13. Lewis TF. Readiness to change, social norms, and alcohol involvement among college students. J Addictions Offender Counseling. $2005 ; 26(1): 22-37$

14. Herman KC, Fahnlander B. A motivational intervention to reduce cigarette smoking among college students: Overview and exploratory investigation. J Coll Counseling. 2003;6(1):46-55.

15. Miech RA, Patrick ME, O'Malley PM, et al. The influence of college attendance on risk for marijuana initiation in the United States: 1977 to 2015. Am J Public Health. 2017;107(6):996-1002.

16. Ward RM, Oswald BB, Galante M. Prescription stimulant misuse, alcohol abuse, and disordered eating among college students. J Alcohol Drug Educ. 2016;60(1):59-80.

17. Iarussi MM. Examining how motivational interviewing may foster college student development. J Coll Counseling. 2013;16(2):158-175.

18. Rollnick S, Miller WR, Butler CC. Motivational interviewing in health care: Helping patients change behavior. New York: Guilford press; 2007. 210 p

19. Hingson RW, Zha W, Weitzman ER. Magnitude of and trends in alcohol-related mortality and morbidity among US college students ages 1824, 1998-2005. J Stud Alcohol Drugs. 2009;Suppl 16:12-20. 